\title{
RETRACTED ARTICLE: Extended rule of five, graphical abbreviated profile, pharmacokinetic and prediction of activity of peptidic HIV-1-PR inhibitors
}

Vishnu Kumar Sahu • Rajesh Kumar Singh •

Pashupati Prasad Singh

Received: 25 February 2010/ Accepted: 25 September 2010/Published online: 19 October 2010

(C) Springer Science+Business Media, LLC 2011

This article has been retracted due to self-plagiarism; a significant proportion of the content was previously published in another journal.

V. K. Sahu $(\bowtie) \cdot$ R. K. Singh · P. P. Singh

Department of Chemistry, Maharani Lal Kunwari Post Graduate

College, Balrampur 271201, UP, India

e-mail: vishnukr_sahu@rediffmail.com 